\title{
NO ROMPERÉ JAMÁS MI ALIANZA CON VOSOTROS (JUE 2,1)
}

\author{
Sor lonel Mihalovici \\ Centro de Estudios Judeo-Cristianos de Madrid
}

\section{RESUMEN}

La Alianza de Dios con los hombres constituye lo esencial de la Revelación. El primer texto que menciona la Alianza es el relato de la Creación, y el último aparece en los Evangelios, cuando Jesús anuncia la Nueva Alianza en su sangre. Pero no son dos Alianzas, la Antigua y la Nueva, sino una y la misma.

Palabras clave: Alianza, Biblia, Creación, Evangelios

\begin{abstract}
God's Covenant with men contitutes the essence of Revelation. The first text to give account of a Covenant is the narration of Creation and the last one appears in the Gospels, when Jesus announces the New Covenant through his blood. Nevertheless, there are not two covenants, an Old one and a New one. In the Bible, the different narrations of covenants are a renewal of the unique Covenant. It's man who is summoned to change his attitude in order to answer, always in a more profound way, to God's call.
\end{abstract}

Key words: Covenant, Bible; Creation, Golspels.

El tema de la Alianza constituye el fondo de toda la Revelación. Se encuentra a cada paso en la Biblia. Es ella que da sentido a la vida de los hombres, porque Dios crea al hombre para llamarle a la Alianza. Se podría definir la Biblia como la expresión de la Alianza de Dios con los hombres.

La alianza es en general una obligación que concierne a dos partes. Se celebra con un juramento como por ejemplo: la alianza entre Abraham y Abimelec en Beersheva (Gn 21,22 ss) Otros ejemplos se pueden êncontrar en Dt 29,9ss y 2 Re 11,4, con un sacrificio como en la ratificación de la Alianza en el Sinaí (Ex 24,4ss) lo que también se recuerda en el Sal 50,5, o con un signo.

Dice el Talmud: Dios habla con palabras humanas, lo que podemos constatar fijándonos en la Alianza. Dios para hacer alianza con los hombres utilizará los términos, los contratos tal como eran conocidos por los pueblos del Próximo Oriente.

En el año 1954 Mendenhall descubrió que existía una gran semejanza entre los tratados de vasallaje hititas y las explicaciones que algunos textos bíblicos dan de la Alianza de Dios con Israel. En un tratado de alianza arameo del siglo VIII a.d. C. entre el rey Maticel de Arpad y el rey de Ktk encontramos: Es dividido este becerro, así sea dividido Maticel y sean divididos sus grandes. (Alusión al rito de pasar en medio de la víctima para sancionar un juramento solemne). En un documento de Mari, se trata de: matar un borriquillo entre (birit) los janeos y las gentes de Idamaraz, con el fin de establecer la paz entre ellos. 
En la Biblia se encuentran dos términos que expresan la Alianza ${ }^{c} e d \hat{u} t$ (testimonio) y $b^{e}$ rît $^{\text {t }}$ (alianza) p.e: luhot ha- ${ }^{c}$ edût o luhot ha- $b^{e}$ rît para designar las tablas de la Ley. La palabra $b^{e}$ rît que se utiliza generalmente para expresar una alianza, tiene un origen incierto. Según unos deriva del asirio birtu (cadena), por ser la alianza un vínculo entre los contrayentes; según otros, de la raíz brit (cortar) de origen hitita. Este segundo sentido lo encontramos p.e. en el libro del Génesis (Gn 15,9ss). Tiene su origen en la costumbre de cortar los animales en dos y pasar por en medio, gesto utilizado por Dios con Abraham en Gn 15,17 ss. En el versículo 18 dice el texto hebreo: Aquel día cortó el Señor una Alianza con Abraham. También en Gn 26,28 Abimelek e Isaac cortan una alianza. En este capítulo encontramos otra etimología de «berit» que es comer, ya que las alianzas se concluyen con una comida: El les dio un banquete y comieron y bebieron. $(26,30)$.

Otros ejemplos de banquete concluyendo una alianza, los encontramos en el libro de Génesis cuando Labán hace un trato con Jacob: Tomaron piedras, hicieron un majano y comieron alli sobre el majano (Gn 31,46) o en el libro del Exodo cuando los 70 ancianos subieron a la montaña con Moisés y Aarón; dice el texto: Vieron a Dios... no extendió El su mano contra los notables de Israel, que pudieron ver a Dios; comieron y bebieron. (Ex 24,11)

A lo largo de los textos bíblicos aparecen muchas alianzas: entre personas, (1Sam 18,3); entre reyes y los súbditos (2Sam 5,3); entre estados (1Re 5,26).

Se encuentran también varias veces un paralelismo entre Paz (Shalom) y Alianza (Berit), porque la alianza debe establecer entre las partes la armonía, el bienestar, la paz. Así en el libro de Josué, a propósito de la alianza con los Gabaonitas, el texto dice: Josué hizo con ellos un pacto (Jos 9,15 ) y en el capítulo 24, la alianza en Siquem aparece como principio de paz y de unidad entre las tribus. En el Primer libro de los Reyes, Salomón hace un pacto con el rey de Tiro: Hubo paz entre Jiram y Salomón, pactando una alianza entre ambos (1Re 5,25). El profeta Ezequiel habla también de la alianza de paz: Yo el Señor he hablado. Concluiré con ellos una Alianza de paz $(\mathrm{Ez} \cdot 34,25)$ y Concluiré con ellos una Alianza de paz, que será para ellos una Alianza eterna (Ez 37,26).

Generalmente la alianza se acompaña de un signo que debe recordar sus obligaciones a los contrayentes. Tenemos varios ejemplos de estos signos: Dijo Abraham (a Abimelek): «estas siete corderas las vas a aceptar de mi mano, para que me sirva de testimonio de que yo he excavado este pozo» (Gn 21,30). Dijo Labán a Jacob: "Aquí está este majano, y aquí está esta estela que he erigido entre nosotros dos. Testigo sea este majano y testigo sea esta estela de que yo no he de traspasar este majano hacía ti, ni tú has de traspasar este majano y esta estela hacía mí para nada malo». (Gn 31,52); lo mismo en el libro de Josué cuando selló el pacto en Siquem: Esta piedra será testigo contra vosotros...

En el relato sacerdotal las tres renovaciones de la Alianza establecida por Dios en tres tiempos claves de la historia de la salvación son: el Shabat, el arco iris y la circuncisión. El Shabat, signo dado desde la Creación y en cuya importancia, en tanto que signo, insiste, por ejemplo, el libro del Exodo: El Sábado es una señal entre Yo y vosotros... (Ex 31,13)

El Arco Iris, después del Diluvio: Pongo mi arco en las nubes, que servirá de señal de la Alianza entre Yo y la tierra. (Gn 9,13)

El signo de la circuncisión: Dijo Dios a Abraham: «Guarda, pues, mi Alianza, tú y tu posteridad de generación en generación. Esta es mi Alianza que habéis de guardar entre Yo y vosotros. Todos vuestros varones serán circuncidados» (Gn 17,1-10). La importancia de este signo está expresada en la Misná en el tratado Ned 3, 11: R. Ismael decia: «la circuncisión es maravillosa, ya que con ella se concluyó trece veces la Alianza». R. Yosé dice: «la circuncisión es maravillosa, ya que puede desplazar el precepto severísimo del sábado..». Rabí decía: «maravillosa es la circuncisión, ya que nuestro padre Abraham, a pesar de que cumplió todos los preceptos, no fue llamado perfecto hasta que no se circuncidó, ya que está escrito: "camina delante mía y sé perfecto» 


\section{LAS ALIANZAS EN LA BIBLIA}

La tradición judía considera ya la Creación como esbozo de la Alianza de Dios con los primeros hombres a quienes Dios da un mandamiento y los asocia a su obra creadora (Gn 1-2).

El segundo escalón es la alianza noaquítica (Gn 9,1-17) Es la Alianza extendida a toda la humanidad e incluso a la creación entera (tierra, animales). Dios impone unas leyes y se compromete a su vez: He aqui que Yo establezco mi alianza con vosotros y con vuestra futura descendencia y con todo ser vivo. (Gn 9,9).

Llegamos a la Alianza con Abraham, que al igual que la Alianza con David pertenece a las «Alianzas de promesa». Porque Abraham obedeció a Dios y siguió sus mandatos, recibe la promesa de la Tierra y de una descendencia. La promesa de la Tierra es esencial en la Alianza con Abraham y se repite en dos relatos. En el primero (Gn 15) Dios responde a la fe de Abraham y se compromete pasando sólo en medio de los animales, en el segundo (Gn 17) le da como signo la circuncisión.

La Alianza con el Patriarca es válida para siempre, incluso si los descendientes pecan, Dios no romperá su promesa: Yo me acordaré de mi Alianza con Jacob, de mi Alianza con Isaac; y recordaré mi Alianza con Abraham; y recordaré la tierra... No los desecharé, ni aborreceré hasta total exterminio, anulando la Alianza con ellos ( $\mathrm{Lv} 26,42.44)$. La misma promesa la encontramos en las palabras de Natán a David: No apartaré de él mi amor (2Sam 7,14). Si Israel peca es castigado pero Dios no rompe su Alianza (Neh 9,18-19; Sal 89,29-35; Lv 26,43). Por ejemplo, Moisés rompe las tablas de la Alianza, pero Dios da unas nuevas.

Hay un texto hitita donde el rey promete una posesión y termina diciendo que si los descendientes pecan, serán castigados, pero nunca la herencia les podrá ser arrebatada.

El nuevo sentido por excelencia en el Primer Testamento, es la Alianza de Dios con Israel en el Sinaí (ver Ex 19ss). De este acontecimiento depende en lo sucesivo toda la religión, o mejor dicho, toda la vida, la existencia de Israel:

- su concepto de Dios;

- sus relaciones con Dios;

- sus relaciones con los demás hombres, tanto como las relaciones de los individuos entre sí, como las del pueblo con las demás naciones.

\section{CONCEPTO DE DIOS Y RELACIONES CON DIOS}

Que un Dios haga una Alianza con un hombre o con un pueblo es un hecho desconocido de todas las religiones de los demás pueblos. Sólo el Dios de Israel llama al hombre a semejante pacto.

Utilizando la forma del antiguo tratado de vasallaje, la Alianza bíblica transforma, sin embargo, por completo la relación del hombre con Dios, porque la Alianza no es una pura protección, ni siquiera una mera elección; es una llamada de Dios a unos hombres para comprometerlos a compartir las responsabilidades con su obra creadora.

A. Neher escribe a este respecto en su obra «Existencia judía»: Dios y el hombre son asociados para construir el mundo y el plan de esta construcción es la Torá. Cuando el hombre no sigue el plan, la construcción se tambalea.

La llamada de Dios es característica de la $b^{e}$ ritt: Dios se dirige a unos hombres y les propone una Alianza con sus obligaciones bien definidas. La conclusión de la alianza está sujeta a la aceptación de estas obligaciones: Así dirás a la casa de Jacob y esto anunciarás a la casa de Israel: "Ya habéis visto lo que he hecho con los egipcios, y cómo a vosotros os he llevado sobre alas de águila y os he traído a mí. Ahora, pues, si de veras escucháis mi voz y guardáis mi Alianza, vosotros seréis mi propiedad personal entre todos los pueblos, porque mía es la 
tierra, seréis para mi un reino de sacerdotes y una nación santa» (Ex 19,3-6). En el capítulo 24, leemos: Tomó (Moisés) después el libro de la Alianza y lo leyó ante el pueblo que respondió: «haremos y escucharemos (nasé venishmá) todo cuando ha dicho el Señor» (Ex 24,7)

Que Israel haya utilizado los antiguos tratados de vasallaje para expresar la Alianza con Dios, confirma el hecho de que la Alianza remonta a la época mosaica, pero sobre todo confirma la realeza de Dios. Dios es el único rey de Israel y esto incluso en la época monárquica, el monarca no es más que un vasallo de Dios, cuyas leyes debe cumplir, con cuya Alianza se debe comprometer: Cuando suba al trono real, deberá escribir para su uso una copia de esta Torá... la llevará consigo; la leerá todos los días de su vida para aprender a temer al Señor su Dios, observando todas las palabras de esta Torá y estos preceptos para ponerlos en práctica (Dt 17,18.19).

Por la Alianza, Dios no es un rey lejano, sino comprometido con su pueblo en la historia de la salvación. Esta historia es como una continua llamada de Dios y una respuesta de los hombres. Y porque es histórica, la Alianza no puede ser estática, sino que necesita una continua renovación; debe ser también la continua búsqueda, la continua respuesta a una llamada siempre nueva y por esto encontraremos tantos relatos de renovación de la Alianza: en Dt 28, 69, renovación de la Alianza en el país de Moab; en Jos 8,32-35, renovación y lectura de la Torá por Josué al oeste de Siquem, entre los montes Garizim y Ebal; Jos 24, 1-28 en Siquem; otra renovación de la Alianza, esta vez hecha por el rey Josías se relata en $2 \mathrm{Re} 23,3$ : El rey se situó en pie junto a la columna y celebró el rito de la alianza ante el Señor: que ellos deberían seguir al Señor y guardar sus mandamientos, sus testimonios y sus preceptos con todo el corazón y toda el alma, y cumplir los términos de esta Alianza tal como estaban en este rollo. Todo el pueblo se comprometió a la Alianza.

Los profetas hablarán de Alianza Nueva, porque la llamada de Dios no permite instalarse cómodamente. Porque existía el peligro de convertir la Alianza en una especie de estatuto, en una regla fija y un deber más bien que una relación viva. Por eso Ezequiel y el segundo Isaías hablan de novedad, creación, renovación libertad (p.e. Is 59,21; 61,8; 66,18ss; Ez 37; 48,35). El profeta Jeremías dice: He aqui que vienen días en los cuales haré nuevo pacto con la casa de Israel y con la casa de Judá... Pondré mi Torá en su interior y sobre sus corazones la escribiré, y Yo seré su Dios y ellos serán mi pueblo... Todos me conocerán, desde el más pequeño hasta el más grande (Jer 31, 31 a.33b.34b). Lo nuevo no es la Alianza sino su interiorización, el don de amor necesario para una vida renovada en la Alianza.

El Amor y la Justicia son dos atributos de Dios que van íntimamente asociados a la Alianza. El término «justicia», aplicado al Dios de la Alianza, debe ser despojado de la carga jurídica que tiene generalmente. Las «acciones justas» del Señor en el canto de Débora (Jue 5,11$)$ son las obras de salvación a favor de Israel. La justicia de Dios es totalmente diferente de la justicia de los hombres. Es una justicia que no puede medirse de acuerdo con conceptos humanos, sino que los que son llamados a su Alianza, tienen que ajustar sus conceptos humanos a los de Dios. El Señor no puede actuar injustamente y los que son llamados a la Alianza deben actuar conforme a la justicia de Dios.

El amor -hesed-relacionado con la Alianza es, no solamente, el amor demostrado en virtud de la Alianza, sino también el amor que da origen a esta Alianza: Pues voy a firmar con vosotros una Alianza eterna: las amorosas y fieles promesas hechas a David (Is 55,3b). André Neher puede decir con razón: La concepción de una alianza entre Dios y los hombres es la contribución más original del pensamiento hebreo a la historia religiosa de la humanidad. En ella se transforma por completo la sensación humana de lo divino, ya que surge en el hombre una opción que nunca ninguna otra revelación divina ha podido proponer; no se trata de religión, ni de veneración, sino de amor. (A. Neher, Existencia judía, p 103) 


\section{RELACIONES ENTRE LOS HOMBRES}

La Alianza crea, entre los que son llamados, una comunidad que influye radicalmente en las relaciones interpersonales. Porque todos los israelitas se consideran presentes al pie del Sinaí, comprometidos en la Alianza, todos son sujetos a las responsabilidades que ésta impone y todos tienen los mismos derechos y los mismos deberes. Todos han sido liberados y ningún israelita podía ser esclavo de otro para siempre. Siete de los Díez Mandamientos, que forman las tablas de la Alianza, hablan de las obligaciones hacía el prójimo.

La tradición judía añade una explicación muy práctica para la vida cotidiana. Se preguntan los sabios por qué en el texto del Exodo se dice que «en este mismo día» vinieron al monte Sinaí. Por qué no «en aquel día», sino en «este». Y contestan porque cada día, cada hombre ha de considerare al pie del Sinaí, escuchando la llamada de Dios, para contestar como Israel lo hizo: cumpliremos todo lo que Dios nos dice. Es la respuesta que desde el jardín del Edén Dios pedía al hombre cuando llamando a Adán le decía: ¿dónde estás? y a cada hombre se le pregunta también ¿dónde estás? con relación a Dios, ¿dónde estás? con relación al prójimo.

\section{QUMRÁN Y LA ALIANZA}

Los que forman parte de la comunidad de Qumrán se consideran los fieles de la «Nueva Alianza» enunciada por los profetas (Jer, $31 ; \mathrm{Ez}, 16$ ).

En la regla de la comunidad los que entran se comprometen a ser fieles a la Alianza: Todos los que entren en la Regla de la Comunidad establecerán una Alianza ante Dios para cumplir todo lo que ordena y para no apartarse de su seguimiento por ningún miedo, terror o aflicción, que suceda durante el dominio de Belial. Cuando entren en la Alianza, los sacerdotes y los levitas bendecirán al Dios de salvación y a todas las obras de fidelidad, y todos los que entren en la Alianza dirán: "Amén, Amén» (1QS Col.1,16-18).

Moisés ordenó un esquema de bendición y maldición: Cuando el Señor tu Dios te haya introducido en la tierra en la que vas a entrar para tomarla en posesión, pondrás la bendición sobre el Monte Garizím y la maldición sobre el Monte Ebal, (Dt 11,29). Josué cumplió esta orden: Se colocaron la mitad en la falda del Monte Garizim y la otra mitad en el Monte Ebal,... Josué leyó todas las palabras de la Ley, la bendición y la maldición..., (Jos 8, 33-34). El mismo esquema se puede encontrar en el ceremonial de entrada en la Alianza de la regla de comunidad. Entresacamos algunas de las palabras pronunciadas por los sacerdotes: Los sacerdotes bendecirán a todos a todos los hombres del lote de Dios que marchan perfectos en todos sus caminos y dirán: "Que os bendiga con todo bien y que os guarde de todo mal. Que ilumine vuestro corazón con la inteligencia de vida y os agracie con conocimiento eterno. Que eleve sobre vosotros el rostro de su gracia para paz eterna»Y Yos levitas maldecirán a todos los hombres del lote de Belial. Tomarán la palabra y diran: «Maldito seas por todas tus impias obras culpables. Que te entregue (Dios) al terror, a manos de los vengadores de venganzas». (1QS Col II 1-6)

Otros textos encontrados en Qumrán, que cantan a Dios en forma de poemas, expresan la felicidad de formar parte de la Alianza, como este pequeño extracto: ¿Cómo podrá nadie alterar tus palabras? Tú, sólo tú, al justo lo has creado. Para él estableciste desde el vientre el tiempo del favor, para que observe tu Alianza y marche por todos (tus caminos), para (volcar) sobre él la multitud de tus misericordias, para abrir toda la estrechez de su alma a la salvación eterna y paz sin fin, sin deficiencias. Sobre la carne tú has alzado su gloria. (1QH Col VII 18-21) 


\section{LA ALIANZA Y EL DIÁLOGO JUDEO-CRISTIANO}

Durante muchos siglos los cristianos se han considerado el Nuevo Israel; el Pueblo de la Nueva Alianza, mientras que la Alianza de Dios con Israel, la mal llamada Antigua Alianza, habría sido abolida y el pueblo de Israel habría sido rechazado y así habría perdido su derecho a la elección.

Muchas persecuciones, injusticias, desprecios, sufrimientos e incluso conversiones forzadas y expulsiones tuvieron por raíz este falso concepto del plan de salvación de Dios.

Terminada la Segunda Guerra Mundial, el mundo horrorizado por el Holocausto ocurrido durante el régimen nazi, comenzó a abrir los ojos y los cristianos a reflexionar sobre la parte de culpabilidad que podría haber tenido una falsa interpretación de las Sagradas Escrituras. Recordamos solamente la muy conocida reunión de Seelisberg.

EI Papa Juan XXIII, que había salvado muchos judíos de ir a los campos de la muerte, nada más llegar al pontificado hizo suprimir de las oraciones del Viernes Santo las palabras «por los pérfidos judíos».

La Declaración Nostra Aetate del Concilio Vaticano II y los Documentos para poner en práctica dicha Declaración, han dado la base a una revisión de muchos conceptos teológicos en relación con el Pueblo de la Alianza.

Juan Pablo II es quien ha dado una vuelta trascendental a esta revisión cuando, el 17 de Noviembre de 1980, en Maguncia, afirmó que Dios nunca abrogó su Alianza con Israel. Y en la sinagoga de Roma, haciendo alusión a los textos: Yo estableceré mi Alianza, una Alianza eterna de ser Yo tu Dios y el de tu posteridad, (Dt 11,29) y: los dones y la llamada de Dios son irrevocables (Rom 11,29), el Pontífice dijo: ... el Dios único, que eligió a Abraham, Isaac y Jacob y estableció con ellos una Alianza de amor eterno, que nunca ha sido revocada. (11 de septiembre de 1987).

Luis Maldonado, en su artículo «El Diálogo Judeo-Cristiano, Implicaciones TeológicoPastorales», publicado en Revista de Espiritualiảad no 237, escribe: Para Juan Pablo II está claro: «Israel sigue siendo el Pueblo de la Alianza. Y así lo ha denominado en su petición de perdón ante el altar de San Pedro y ante el Muro Occidental».

En el año 2001, la Pontifica Comisión Bíblica, publicó el documento «El Pueblo Judío y sus Escrituras Sagradas en la Biblia Cristiana» que demuestra también que la Palabra de Dios une judíos y cristianos en una misma Alianza vivida cada uno según su tradición.

\section{UNA SOLA ALIANZA}

En el profeta Jeremías 31, 31-34 encontramos, según el exegeta N. Lohfing, «el trasfondo de los enunciados neotestamentarios sobre la Nueva Alianza». Y aquí surge la pregunta: ¿hay dos alianzas, una antigua y otra nueva? Sin embargo, leyendo el texto con más atención vemos que es el mismo Dios, y la misma Torá, la misma Palabra de Dios que llama a todo hombre a su Alianza: Yo seré su Dios y ellos serán mi pueblo. Lo que va a cambiar es el hombre y su respuesta a esta llamada. Cuando durante su Ultima Cena, Jesús habla de la Nueva Alianza en su sangre, recoge la tradición profética que afirma la renovación de la Única Alianza, que como recuerda Juan Pablo II, nunca ha sido derogada.

Dios llama a todos los creyentes, judios y cristianos, a llevar su Nombre y a publicar su gloria en todo el Universo. 